\title{
Inserm Propionibacterium acnes CAMP factor 1 recognized Toll-Like Receptor-2
}

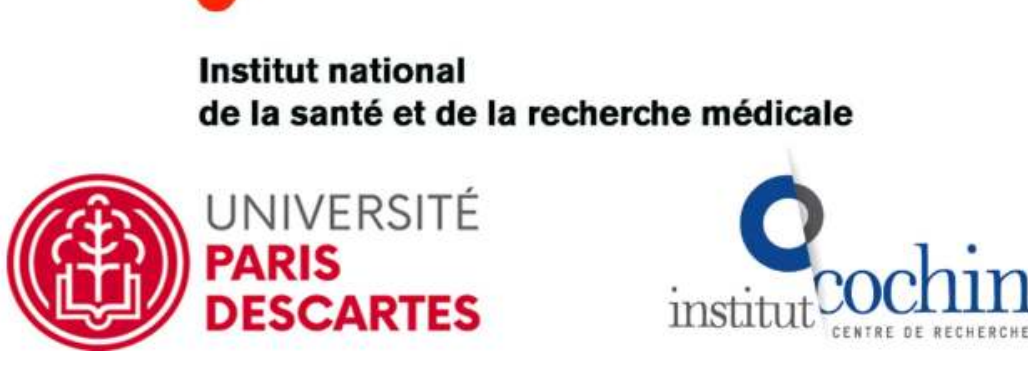

Grange $\mathrm{PA}^{1}$, Lheure $\mathrm{C}^{1,2}$, Ollagnier $\mathrm{G}^{1}$, Morand $\mathrm{P}^{3}$, Désiré $\mathrm{N}^{4}$, Sayon $\mathrm{S}^{4}$, Corvec $\mathrm{S}^{5}$, Marcelin $\mathrm{AG}^{4}$, Dreno $\mathrm{B}^{6}$, Dupin $\mathrm{N}^{1,2}$.

1Université Sorbonne Paris Descartes, Faculté de Médecine, INSERM, U1016, Laboratoire de Dermatologie, Paris, France, France; ${ }^{2}$ AP-HP, Hôpital Cochin-Pavillon Tarnier, Service de Dermatologie-Vénéréologie, Paris, France; ${ }^{3}$ APHP, Groupe Hospitalier Paris Centre Cochin-Hôtel Dieu-Broca, Service de Bactériologie-CNR Streptocoques, Paris, France; ${ }^{4}$ AP-HP, Groupe hospitalier Pitié Salpêtrière, Laboratoire de Virologie, Paris, France; 5 Service de Bactériologie-Hygiène hospitalière, CHU de Nantes, Nantes; ${ }^{6} \mathrm{CHU}$, Service de dermatologie, CIC, Hôtel Dieu, Nantes, INSERM U 892, CNRS U6299, Hôtel Dieu, Nantes, France

ABSTRACT

Propionibacterium acnes $(P$. acnes) is a major skin-associated bacterium that opportunistic pathogen involved in several invasive infections. $P$. acnes induced strong immune response involving lipoteicoic acid and peptidoglycan but no $P$. acnes surface protein interacting with Toll-like receptors have been identified. $P$. experiments and two proteins of 24.5 - and $27.5-\mathrm{k}$ Da were specifically recognized
by TLR2 and subsequently characterized as CAMP factor 1 by LC-MS/MS. Purified CAMP factor 1 induce IL-8 mRNA and protein production by activating IL-8 gene promoter, and using antibodies against TLR2 significantly decrease the and II types strains associated with a capacity to produce large amount of IL-8, than strains typed IA1 and IA2 associated with low IL-8 production. Furthermore, CAMP factor 1 could be divided into two major distinct genetic groups corresponding with highly polymorphic sequence from strains with moderate and high CAMP1-TLR2 binding activities; and with low polymorphic sequences fro intensity.

\section{OBJECTIVES}

The surface proteins of $P$. acnes involved in the activation of TLR2 on keratinocytes and monocytes and leading to inflammatory on keratinocytes and monocytes and leading to inflammatory
lesions in acne have yet to be identified. The aim of this study was
to identify surface proteins of $P$. acnes recognized by TLR2 in several strains with different inflammatory profiles.

\section{METHODS}

Bacterial culture and protein extraction. 27 strains of $P$. acnes were used (from ATCC, the
Dermatology Department of Nantes Hospital, and the Bacteriology Department of Cochin Hospital)

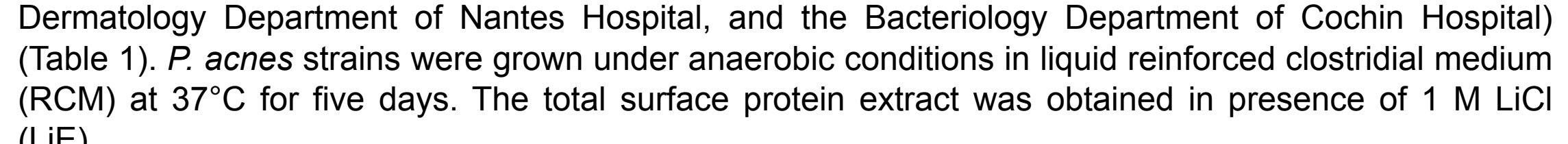
Cell culture and stimulation. The human keratinocyte HaCaT and the monocyte ThP1 cells lines were
grown in DMME and RPII, respectively. Cels were incubated with a $P$. acnes suspension adjusted to
the appropriate concentration $(620$ nm) or with LiE. DNA extraction and phylotyping. DNA was extracted from bacterial strains with the E.Z.N.A.
BBacterial DNA kit. Phylotyping was performed by multiplex touchdown PCR [Barnard 2015].

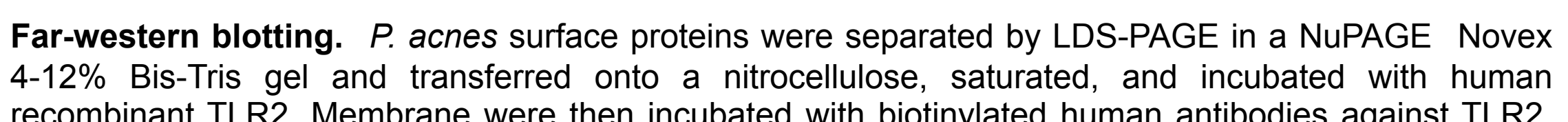

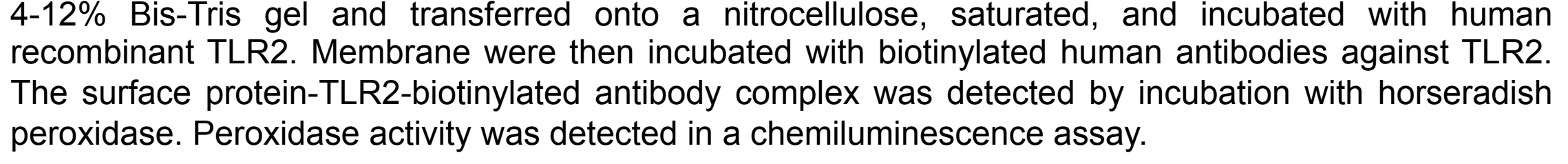
Protein elution. Proteins were fractionated by $12 \%$ SDS-PAGE and excised protein bands were
incubated for $18 \mathrm{~h}$ at $37^{\circ} \mathrm{C}$ in $1 \%$ SDS in PBS. Excess of SDS was removed by cold acetone
precipitation Protein pellets were suspended in PBS and protein concentration was determined at 280 Cell transfection and luciferase reporter gene assay. HaCaT were transfected with reporter

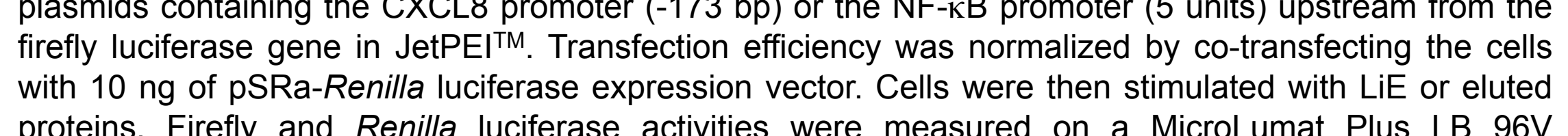

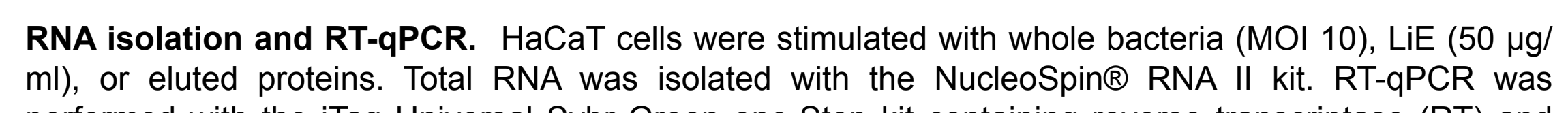
performed with the iTaq Universal Sybr Green one-Step kit containing reverse transcriptase (RT) and
Tago polymerase. Complementary DNA and standard PCR amplification were carried out on a
Lighthycler Nano. The amount of RNA in stimulated cells relative to control cells was calculated by the LC-MSIMS characterization of proteins of interest. Proteins were separated by $10 \%$ SDS-PAGE and
proteins recognized by TLR2 were matched to stained bands, excised from the gel, reduced and proteins recognized by 2 RR2 were matched to stained bands, excised from the gel, reduced and
alkylated. Trysin digestion was performed and peppide extracts were enriched, separated and analyzzed
with a 6520 Accurate-Mass Q-TOF LCIMS machine equipped with an HPLC-chip cube interface. The

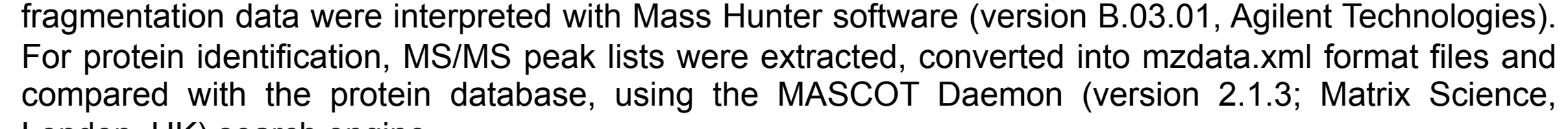

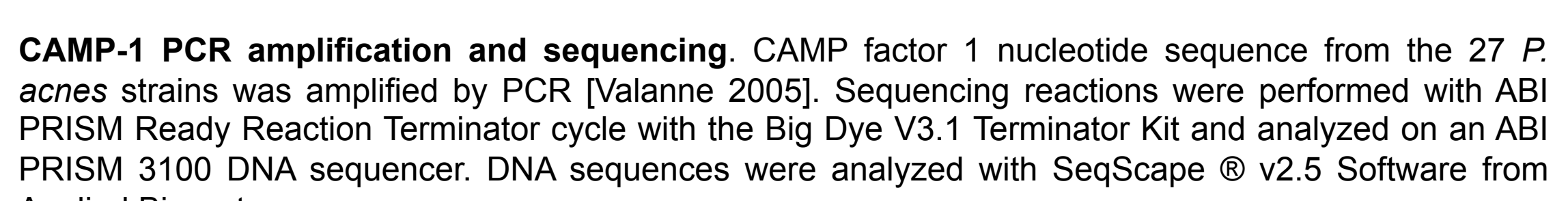

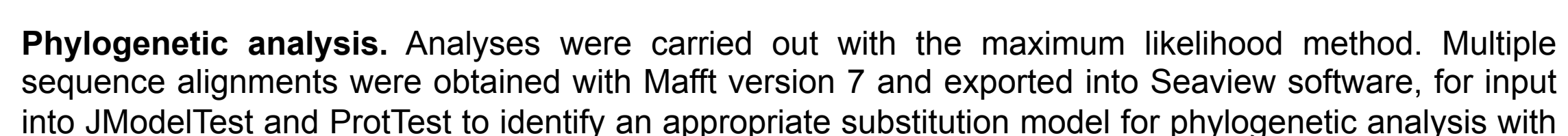

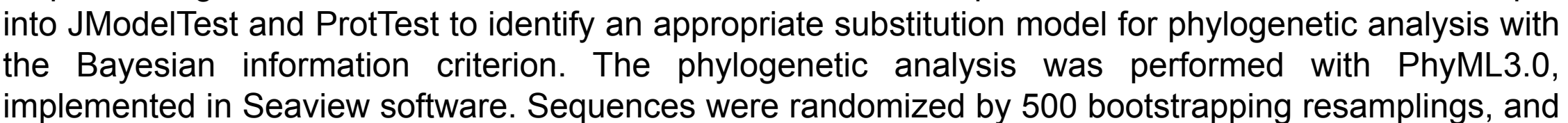
implemented in Seaview software. Sequences were randomized by 50
statisitical analyses were performed on 100 datasets for each analysis

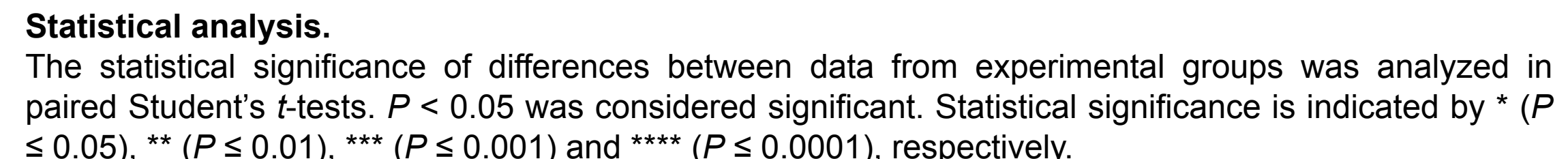

Table 2 : Comparison of $P$. acnes inflammatory capabilities with phylogroup and CAMP factor 1 gene nucleotide sequence

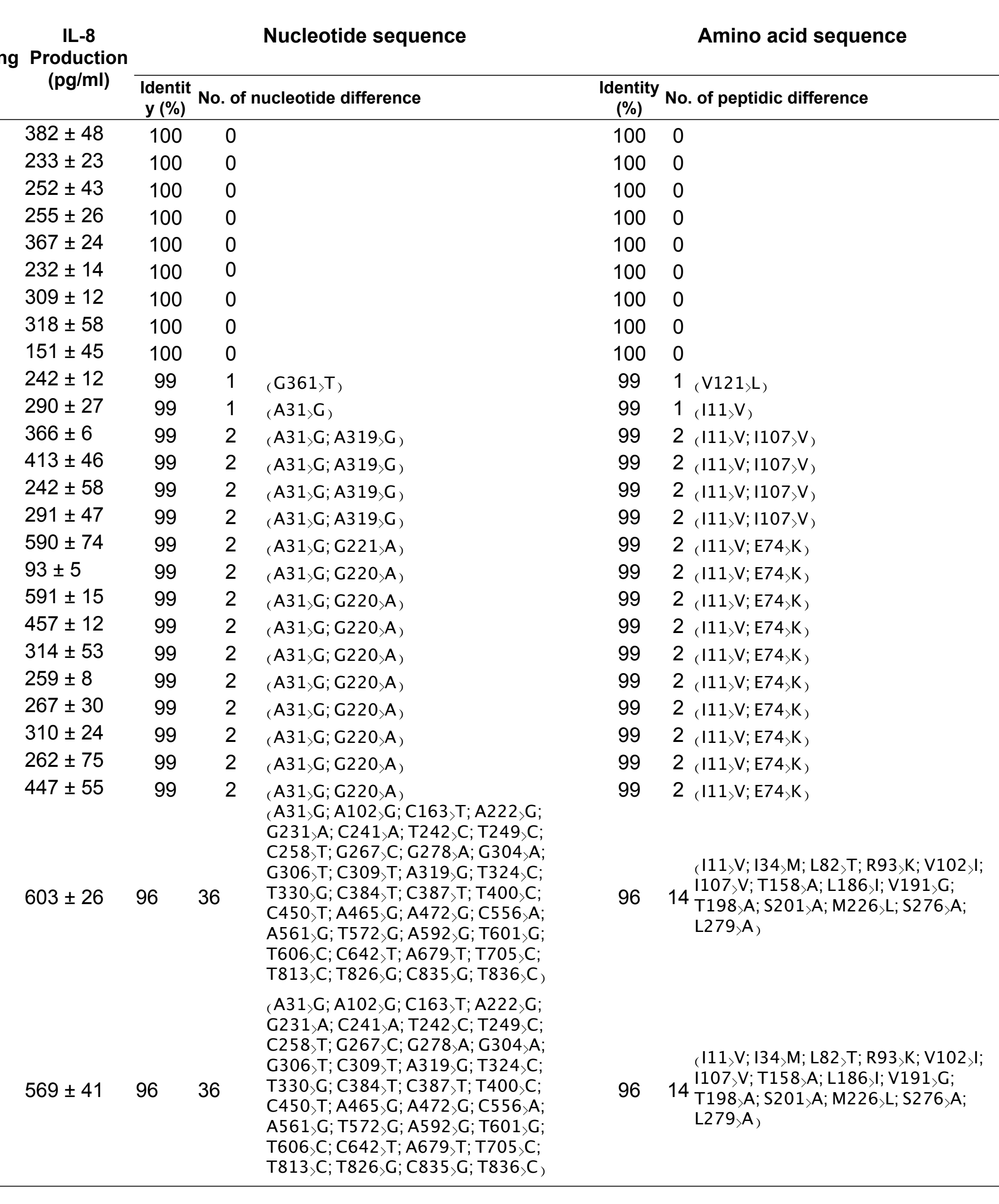

REFERENCES

Valanne, et al., (2005) Microbiology. 151:1369-1379
Barnard et al, (2015) J. Clin. Microbiol : 53:1149-1155.

Barnard et al., (2015) J. Clin. Microbiol. 53:1149-1155.
Lheure, et al., (2016) PLoS ONE. (11): e0167237. doi:10.1371/journal.pone.0167237

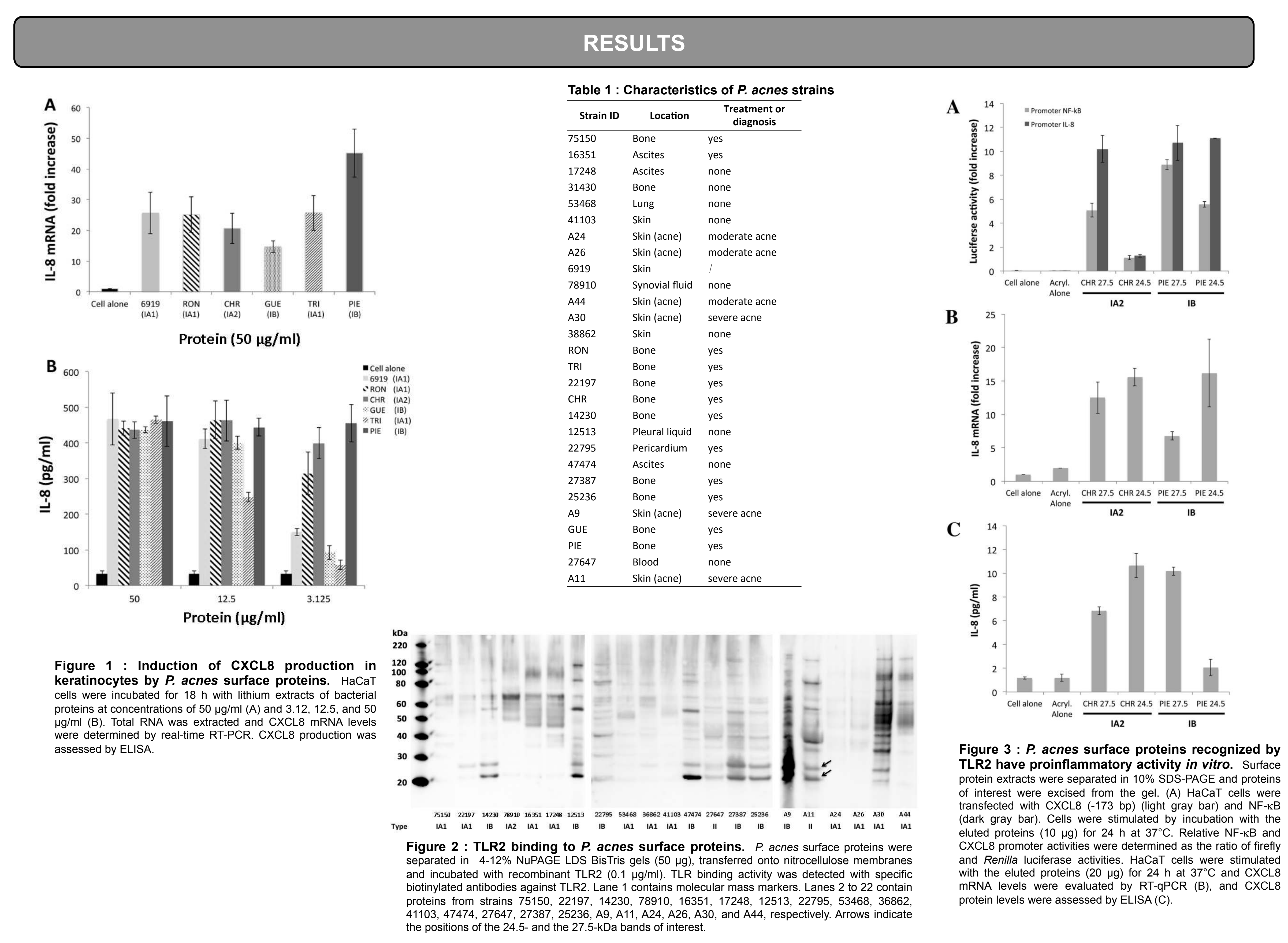

Keratinocytes were stimulated with LiE for $18 \mathrm{~h}$ and representative results are shown for the evaluation of the CXCL8 production from the $27 P$. acnes strains used in this study (Fig 1 , extract are a suitable starting material for identification of the surface protein recognized by TLR2. Far-western blotting was performed on total LiE, and two protein bands with apparent molecular masses of 24.5 - and 27.5-kDa were recognized by TLR2 (Fig 2). Eluted 24.5- and 27.5-kDa proteins were able to activate the NF-kB and CXCL8 promoters (Fig. 3A),
to induce 10 - to 100 -fold induction of $C X C L 8$ transcription (Fig. 3B) as well as CXCL8 protein production to $10 \mathrm{pg} / \mathrm{ml}$ ( Fig 3C). As the 24.5-kDa and 27.5-kDa proteins appeared to be to induce 10 - to $100-$-fold induction of CXCL8 transcription (Fig. $3 B$ ) as well as CXCL8 protein production to $10 \mathrm{pg} / \mathrm{ml}$ ( Fig $3 C)$
recognized by TLR2 in $48 \%$ of the strains tested, we chose to focus on the characterization of these two proteins.

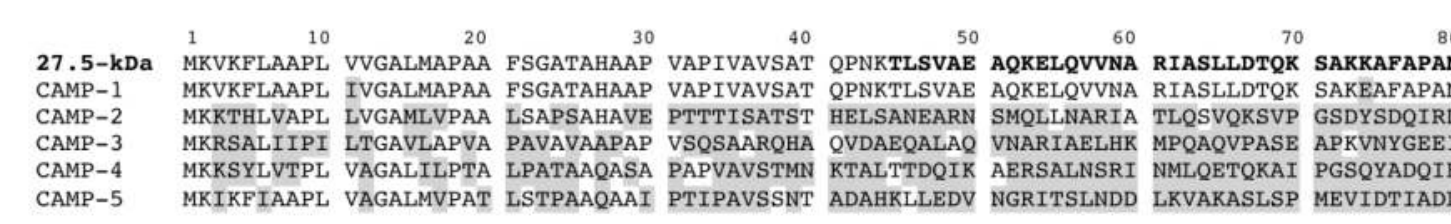

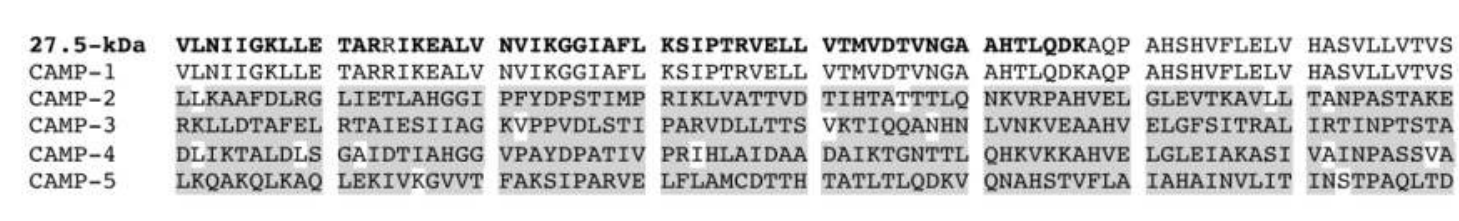 $\mathrm{w}$

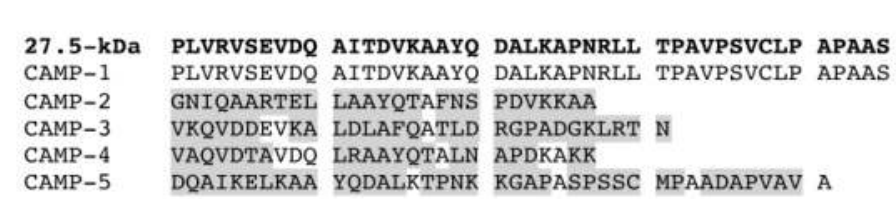

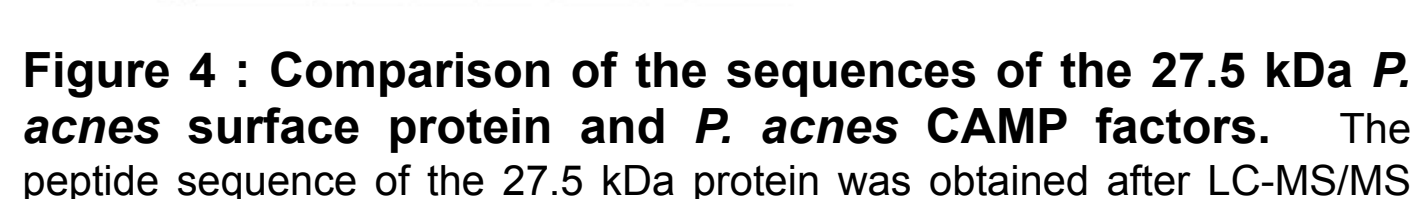

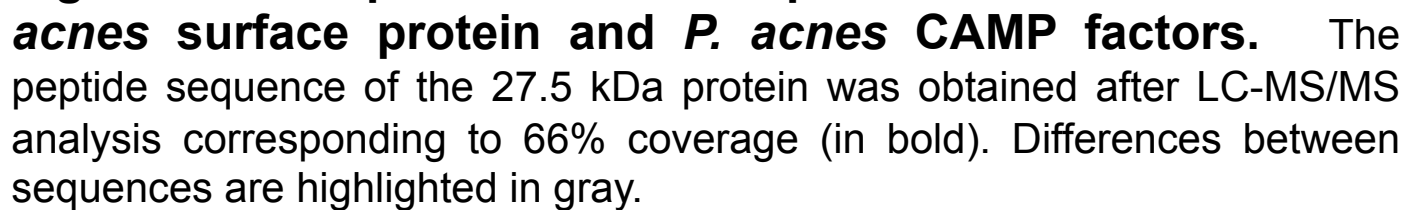

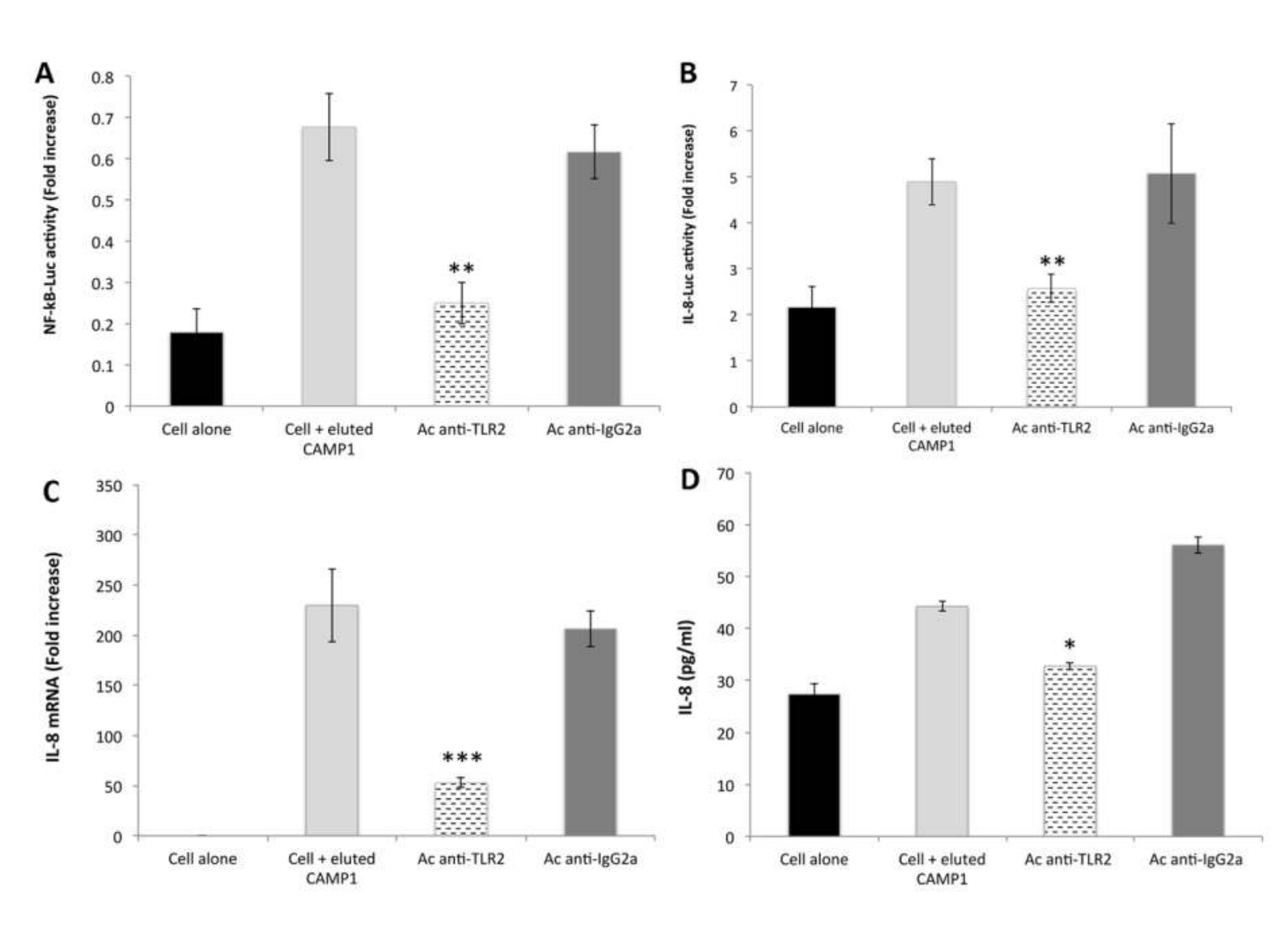

Figure 5: TLR2 blockade inhibits $P$, acnes CAMP factor

Proteins of interest were characterized by LC-MS/MS and protein sequence database searches identified the two proteins of interest as products of gene encoding a hypothetical 285-

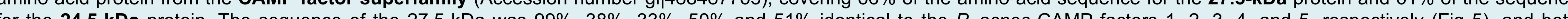
proteins corresponded to the same $P$. acnes surface protein. The stimulation of keratinocytes with eluted CAMP-1 protein after their prior treatment with anti- $-\mathrm{LR} 2$ antibodies resulted in significantly lower levels of NF-KB $(P=0.008)$ and $C X C L 8(P=0.0012)$ promoter activation (Fig $5 \mathrm{~A}, \mathrm{~B})$, and, consequently, significantly lower levels of $C X C L 8 \mathrm{mRNA}(P=0.00047)$ (Fig
$5 \mathrm{C}$ ) and $C X C 18$ protein production in keratinocytes $(P=0.0024)$ (Fig 5D). No such effect was observed when this experiment was performed with the lgG isotype control. Thus, CAMP $5 \mathrm{C})$ and $C X C L 8$ protein production in keratinocytes $(P=0.0024$ ) (Fig $5 D)$. No such effect was observed when this experiment was performed with the IgG isotype control. Thus, CAMP
factor 1 isolated from $P$. acnes interacts with TLR2, triggering a downstream signaling pathway leading to $C X C L 8$ production.

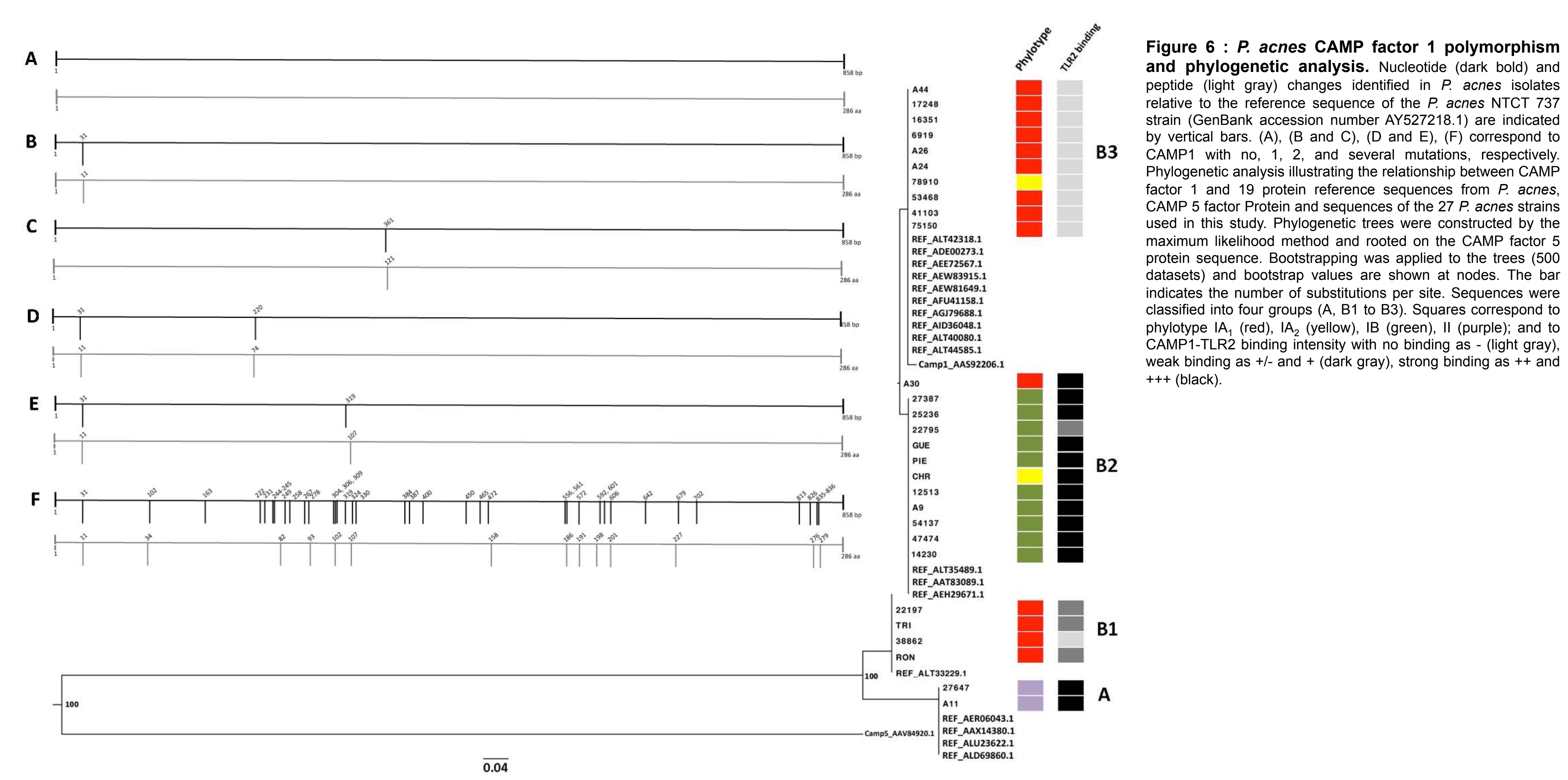

We identified four different phylotypes: IA 1 (15 strains), IA 2 (2 strains), IB (9 strains) and II (2 strains) (Table 2) with most of the strains of types IB and II having strong TLR2 binding activity and producing high level of $\mathrm{CXCL8}$ (Table 2).
CAMP factor $1 \mathrm{DNA}$ sequences were then classified into 4 groups according to the number of nucleotide polymorphisms. Group A corresponded to CAMP factor 1 gene with no nucleotide polymorphisms. Group $F$ corresponded to CAMP factor 1 genes with sequences containing 36 single nucleotide polymorphisms (Fig 6, Table 2). Strains with weaker TLR2 binding activity were found to have CAMP factor 1 genes with no nucleotide polymorphism or sequences with 1 or 2 nucleotide polymorphisms, corresponding to $|11>V| 1107>$,$V and$ Vid large numbers of nucleos Phylogenetic analysis of the CAMP factor 1 sequences revealed the existence of two distinct genetic groups supported by high bootstrap values, and corresponding to strains
phylotyped II with CAMP factor 1 containing 36 nucleotide polymorphisms (group A), and to the strains phylotyped IA, IA, IB with CAMP factor 1 containing no nucleotide
polymorphism, one nucleotide polymorphism, and 2 nucleotide polymorphisms (Group B) (Fig 6; Table 2). Group B contained three genetically related clusters (B1, B2, B3) corresponding to CAMP1 very weakly (B1) strongly (B2) and not recognized (B3) by TLP2

\section{CONCLUSIONS}

Our findings indicate that CAMP factor 1 may contribute to $P$. acnes virulence, by amplifying the inflammation reaction through direct interaction with TLR2. 